\title{
EMBOLIZAÇÃO ARTERIAL SELETIVA EM FÍSTULA ARTERIOVENOSA UTERINA PÓS-TRAUMÁTICA*
}

\author{
Omero Benedicto Poli-Neto ${ }^{1}$, João Batista Víbrio Neto ${ }^{2}$, Antonio Alberto Nogueira ${ }^{3}$, \\ Francisco José Candido dos Reis ${ }^{3}$
}

\begin{abstract}
Resumo Malformações arteriovenosas uterinas são pouco freqüentes. Os autores relatam um caso de fístula arteriovenosa traumática tratada por embolização seletiva das artérias uterinas, método que tem sido utilizado no controle da hemorragia pós-parto e hemorragia resultante de malignidade pélvica. Uma discussão é apresentada para ressaltar uma conseqüência da perfuração uterina e o controle da hemorragia sem sacrificar a fertilidade.

Unitermos: Útero; Embolização seletiva; Malformação arteriovenosa; Hemorragia pós-aborto.
\end{abstract}

Abstract Selective arterial embolization of uterine post-traumatic arteriovenous fistula

Uterine arteriovenous malformations are uncommon. The authors report a case of traumatic arteriovenous fistula treated by selective uterine arteries embolization. Selective artery embolization has been effectively used to control postpartum hemorrhage and hemorrhage resulting from pelvic malignancy. A discussion on the consequences of uterine perforation and the control of hemorrhage without sacrificing fertility is presented.

Key words: Uterus; Selective embolization; Arteriovenous malformation; Postabortal hemorrhage.

\section{INTRODUÇÃO}

As hemorragias após aborto, na maioria das vezes, são conseqüentes à presença de restos ovulares na cavidade uterina. Nestes casos, nova curetagem uterina controla o sangramento e evita maiores complicações $^{(\mathbf{1})}$. Menos freqüentemente, as hemorragias após aborto resultam de anormalidades vasculares, congênitas ou adquiridas. A maioria das publicações consiste de relatos de caso ou série de casos e experiências individuais, sendo que, no passado, grande número desses casos era diagnosticado e tratado por histerectomia, comumente realizada devido a sangramento refratário ou exacerbado pela curetagem uterina $^{(2)}$. Recentemente, a embolização arterial seletiva tem emergido como técnica percutânea efetiva para controle do san-

* Trabalho realizado no Hospital das Clínicas da Faculdade de Medicina de Ribeirão Preto - Universidade de São Paulo (FMRP-USP), Ribeirão Preto, SP.

1. Médico Assistente do Departamento de Ginecologia e Obstetrícia do Hospital das Clínicas da FMRP-USP.

2. Médico Assistente do Departamento de Radiologia do Hospital das Clínicas da FMRP-USP.

3. Professores Doutores do Departamento de Ginecologia e Obstetrícia do Hospital das Clínicas da FMRP-USP.

Endereço para correspondência: Prof. Dr. Francisco José Can dido dos Reis. Departamento de Ginecologia e Obstetrícia FMRP-USP. Avenida Bandeirantes, 3900, 8ํandar. Ribeirão Preto, SP, 14049-900. E-mail: fjcreis@fmrp.usp.br

Recebido para publicação em 4/8/2003. Aceito, após revisão, em 19/9/2003. gramento genital agudo e crônico em distúrbios obstétricos e ginecológicos ${ }^{(3)}$.

Relatamos, neste artigo, um caso clínico de fístula arteriovenosa traumática tratado com sucesso pela embolização arterial seletiva. O objetivo do relato é ressaltar a possibilidade do diagnóstico de malformação arteriovenosa nos sangramentos anormais pós-curetagem e difundir a embolização seletiva como método conservador, seguro e efetivo no tratamento dessas pacientes.

\section{RELATO DO CASO}

Uma jovem com idade de 21 anos, primigesta e com atraso menstrual de dez semanas, deu entrada no nosso serviço com diagnóstico clínico de aborto incompleto. Ela foi tratada por curetagem uterina, tendo como intercorrência perfuração uterina ao final do procedimento. A evolução clínica foi boa e a paciente teve alta no primeiro dia pós-operatório. Ela procurou o serviço, novamente, no segundo e no terceiro mês após o tratamento inicial, com sangramento vaginal moderado. $\mathrm{O}$ exame clínico da pelve era normal em ambas as datas. A paciente não tinha antecedentes de fluxos menstruais anormais e fizera uso de acetato de medroxiprogesterona de depósito alguns dias após a curetagem. Du- rante a última admissão os exames evidenciaram nível de hemoglobina de 7,9 g/dL e hematócrito de $24 \%$.

Foi realizada ultra-sonografia pélvica, que identificou uma área com imagens hipoecóicas, tubulares e coalescentes, medindo $1,5 \mathrm{~cm}$, envolvendo a porção intramural e submucosa do miométrio na parede anterior do corpo uterino, com intenso fluxo de baixa resistência ao Doppler (Figura 1A). O endométrio era fino, ecogênico e com limites imprecisos junto à lesão descrita. A arteriografia evidenciou hipertrofia da artéria uterina direita, com presença de múltiplas pequenas fístulas arteriovenosas de fluxo lento em topografia miometrial (Figura 1B). Foi então realizada embolização de ambas as artérias uterinas com torpedos de Gelfoam, com excelente resultado angiográfico (Figura 1C).

A paciente evoluiu com discreto hematoma no local da punção femoral e recebeu alta no primeiro dia após o procedimento, sem sangramento genital e sem necessidade de analgésico. Após o procedimento, não apresentou recorrência do sangramento.

\section{DISCUSSÃO}

As malformações arteriovenosas uterinas são bastante incomuns. Estas lesões 


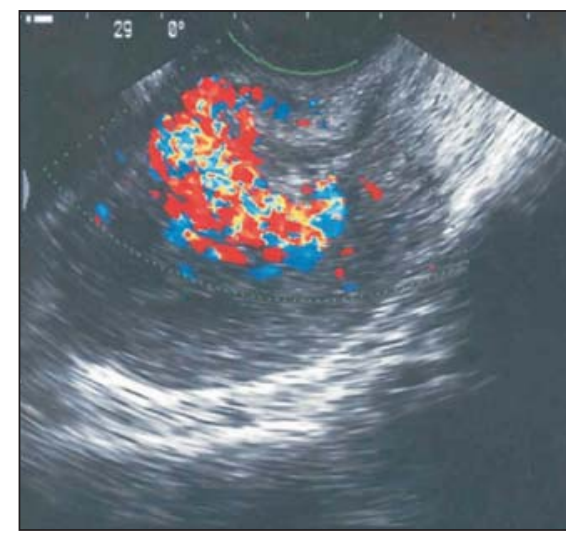

A

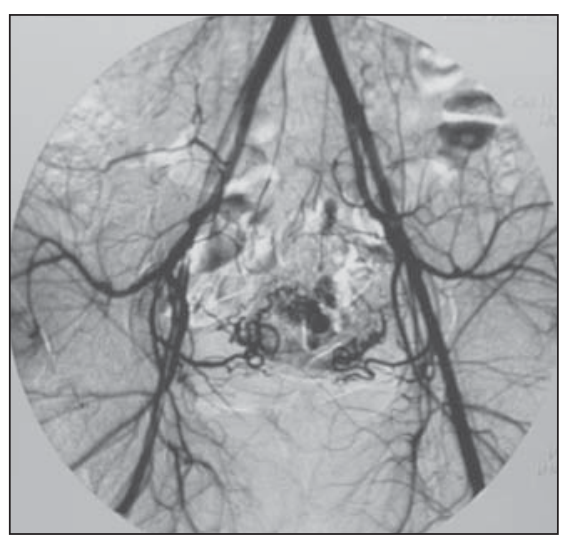

B

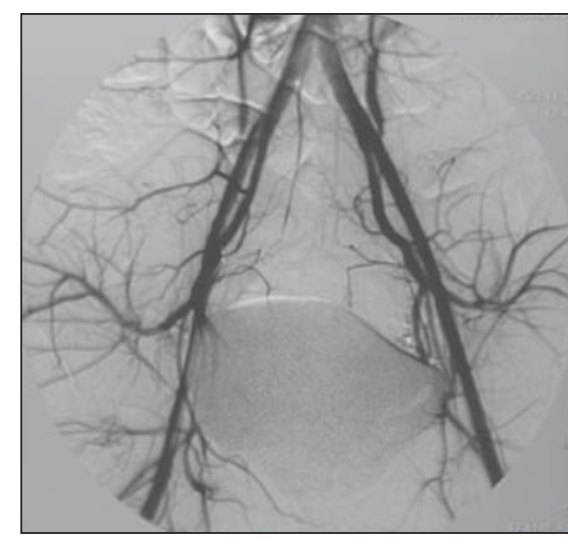

C

Figura 1. A: Ultra-sonografia transvaginal e Doppler evidenciando imagem sugestiva de fístula arteriovenosa intramiometrial. B: A arteriografia confirma a presença da fístula e demonstra extravasamento do meio de contraste. C: Aspecto imediato após a embolização seletiva das artérias uterinas.

podem ser congênitas ou adquiridas, secundárias a trauma, cirurgia, neoplasia e infecção ${ }^{(2,4)}$.

Fleming et al., em 1989, relataram, num período de dez anos, seis casos de anormalidades vasculares uterinas clinicamente significantes e, destes, quatro casos tinham história prévia de ao menos uma curetagem uterina $^{(2)}$. Kwon e Kim, em 2002, relataram 24 casos de anormalidades vasculares uterinas iatrogênicas diagnosticados em oito anos, dos quais 21 pacientes tinham história prévia de pelo menos uma curetagem uterina ${ }^{(\mathbf{5})}$. O surgimento dessas alterações vasculares após complicações da curetagem, como a perfuração uterina, é possível, devido à proximidade entre plexo venoso e artérias arqueadas, veias e artérias radiais e espiraladas ${ }^{(\mathbf{6})}$.

No passado, o diagnóstico era geralmente feito após a histerectomia e o exame anatomopatológico ${ }^{(2)}$. Atualmente, o diagnóstico é sugerido pela ultra-sonografia associada ao método Doppler ${ }^{(7)}$, mas deve ser confirmado pela arteriografia. As imagens mais sugestivas à ultra-sonografia são alterações císticas ou tubulares na parede uterina, com fluxo sanguíneo arterial de alta velocidade e baixa resistência ao Doppler $^{(5)}$.

A curetagem não é terapêutica nesses casos e pode exacerbar o sangramento ${ }^{(8)}$. Apesar de alguns autores relatarem a possibilidade de seguimento clínico com regressão espontânea da fístula arterioveno$\mathrm{sa}^{(8)}$, algumas vezes o volume do sangramento e o quadro clínico da paciente exigem conduta emergencial. A embolização de vasos uterinos para tratamento de sangramentos após partos ${ }^{(\mathbf{9 , 1 0})}$ e após aborto ${ }^{(\mathbf{1})}$ já vem sendo usada desde as décadas de 70 e 80 do século passado. A embolização arterial transcateter é considerada segura e efetiva no tratamento dessas condições ${ }^{(\mathbf{5})}$, e a paciente pode aguardar o retorno dos ciclos menstruais mensais sem repercussões significantes na fertilidade dois a cinco meses após o procedimento ${ }^{(11)}$.

O uso de partículas de Gelfoam, no presente caso, foi escolhido devido à idade da paciente e à sua condição de nulípara, pois o Gelfoam proporciona oclusão apenas temporária dos vasos, podendo possibilitar o fechamento das lesões por trombose devido à interrupção ou redução do fluxo arterial. A embolização bilateral é importante nestes casos, devido à extensa circulação colateral através da artéria uterina contralateral. Por se tratar de material reabsorvível, as artérias retornam ao normal algumas semanas após a oclusão com Gelfoam, não havendo, portanto, risco associado de infertilidade ${ }^{(\mathbf{1 1 , 1 2})}$. Se houver recorrência, existe ainda a possibilidade de novo tratamento endovascular seletivo com utilização de agentes de emboligênicos definitivos.

Embora a embolização seletiva das artérias uterinas provavelmente não interfira na fertilidade, é possível haver maior risco de complicações durante gravidez subseqüente, como apresentação anômala, parto pré-termo, maior índice de cesárea e hemorragia pós-parto ${ }^{(13)}$. A boa aceitação do método pelas pacientes também já foi observada na literatura, devido às poucas complicações $^{(\mathbf{1 4 , 1 5 )}}$. Neste nosso caso houve apenas um pequeno hematoma no sítio da punção femoral. A dor é o sintoma mais comum após a embolização e, em geral, é mais intensa quando há isquemia de grandes massas, como tumores malignos ${ }^{(16)}$. Além disso, o custo do procedimento é significativamente inferior ao da histerectomia $^{(17)}$. Dessa forma, sugerimos que o diagnóstico de malformação vascular uterina seja aventado quando há sangramento anormal após curetagem.

$\mathrm{O}$ rastreamento com ultra-sonografia e Doppler deve preceder uma nova curetagem. Diante de suspeita ultra-sonográfica, a arteriografia pode confirmar o diagnóstico e promover o tratamento da fístula por intermédio da embolização seletiva das artérias uterinas, com benefícios evidentes para a paciente.

Este procedimento é ainda pouco utilizado $^{(3)}$, e o objetivo deste relato é enfatizar seu papel como método altamente efetivo no controle do sangramento genital, sendo de rápida realização, baixo custo, pouco invasivo, apresentando índices reduzidos de complicações.

\section{REFERÊNCIAS}

1. Haseltine FP, Glickman MG, Marchesi S, Spitz R, Dlugi A, DeCherney AA. Uterine embolization in a patient with postabortal hemorrhage. Obstet Gynecol 1984;63:78-80.

2. Fleming H, Ostor AG, Pickel H, Fortune DW. Arteriovenous malformations of the uterus. Obstet Gynecol 1989;73:209-14.

3. Vendantham S, Goodwin SC, McLucas B, Mohr G. Uterine artery embolization: an underused method of controlling pelvic hemorrhage. Am J Obstet Gynecol 1997;176:938-48. 
4. Ghosh T. Arteriovenous malformation of the uterus and pelvis. Obstet Gynecol 1986;68:40-3.

5. Kwon JH, Kim GS. Obstetric iatrogenic arteria injuries of the uterus: diagnosis with US and treatment with transcatheter arterial embolization. RadioGraphics 2002;22:35-46.

6. Levi CS, Holt SC, Lyons EA, Lindsay DJ, Dashefsky SM. Normal anatomy of the female pelvis. In: Callen PW, ed. Ultrasonography in obstetrics and gynecology. 4th ed. Philadelphia: Saunders, 2000 781-813

7. Polat P, Suma S, Kantarcý M, Alper F, Levent A Color Doppler US in the evaluation of uterine vascular abnormalities. RadioGraphics 2002;22:4753.

8. Flynn MK, Levine D. The noninvasive diagnosis and management of a uterine arteriovenous malfor- mation. Obstet Gynecol 1996;88:650-2.

9. Heaston DK, Mineau DE, Brown BJ, Miller FJ Jr. Transcatheter arterial embolization for control of persistent massive puerperal hemorrhage after bilateral surgical hypogastric artery ligation. AJR 1979; $133: 152$

10. Pais SO, Glickman M, Schwartz P, Pingoud E, Berkowitz R. Embolization of pelvic arteries for control of postpartum hemorrhage. Obstet Gynecol 1980;55:754-8.

11. Stancato-Pasik A, Mitty HA, Richard HM III, Eshkar N. Obstetric embolotherapy: effect on menses and pregnancy. Radiology 1997;204:791-3.

12. Pelage JP, LeDref O, Mateo J, et al. Life-threatening primary postpartum hemorrhage: treatment with emergency selective arterial embolization. Radiology 1998;208:359-62.
13. Goldberg J, Pereira L, Berghella V. Pregnancy after uterine artery embolization. Obstet Gynecol 2002;100:869-72.

14. Salerno S, Belli AM. Percutaneous treatment of uterine fibroleiomyomas: analysis of complications and quality of life after embolization. Radiol Med (Torino) 2001;101:360-4.

15. Zupi E, Pocek M, Dauri M, et al. Selective uterine artery embolization in the management of uterine myomas. Fertil Steril 2003;79:107-11.

16. Yalvac S, Kayikcioglu F, Boran N, et al. Embolization of uterine artery in terminal stage cervical cancers. Cancer Invest 2002;20:754-8.

17. Al-Fozan H, Dufort J, Kaplow M, Valenti D, Tulandi T. Cost analysis of myomectomy, hysterectomy, and uterine artery embolization. Am J Obstet Gynecol 2002;187:1401-4. 